\title{
Les microsystèmes électromécaniques optiques
}

\author{
Alain BOSSEBOEUF, Filippo FABBRI, Fabien PARRAIN, Philippe COSTE, Hervé BERTIN et Wei XU \\ Institut d'Électronique Fondamentale, CNRS-Université Paris Sud \\ alain.bosseboeuf@u-psud.fr
}

Depuis le début des années 80 , naissance des premiers systèmes miniaturisés associant des microsystèmes électromécaniques (MEMS) en technologie silicium et de l'optique, les fonctions optiques réalisées, les technologies de fabrication et d'intégration employées et les applications potentielles des microsystèmes électromécaniques optiques (MEMS optiques) et des microsystèmes optoélectromécaniques (MOEMS) se sont très largement étendues et diversifiées. Des contributions à ces évolutions sont illustrées ici par quelques exemples de projets en cours dans notre laboratoire.

$\mathrm{L}$ es travaux récents menés en France et à l'étranger visent à la fois un contrôle spatial et spectral de la lumière, un contrôle de la polarisation et/ou de la focalisation, l'étude de micro ou nano résonateurs avec couplage optomécanique, l'exploitation de micro et nano composants optiques de tous types (passifs, actifs, réfractifs et diffractifs, guidés, métamatériaux) et l'introduction de I'optofluidique. Concernant les technologies d'intégration, l'intégration des MEMS optiques ou MOEMS avec de l'électronique CMOS, l'intégration des sources et détecteurs optoélectroniques et le développement de technologies de packaging sont des challenges permanents. Des efforts importants sont aussi menés dans les laboratoires pour mélanger les technologies de fabrication silicium, verre, III$V$ et polymères (intégration hétérogène), pour réaliser des matrices de MOEMS par intégration 3D hybride (multi-puces/multiwafers) ou pour développer des microbancs optiques.

Enfin, même si le marché reste encore très largement dominé par les domaines de l'affichage et des télécommunications, les MEMS optiques et les MOEMS couvrent maintenant un très large champ d'applications potentielles dans d'autres secteurs : médical et biomedical, industrie, aéronautique, etc.

\section{Intérêt et bref historique des microsystèmes optiques}

Les microsystèmes électromécaniques (MEMS) sont des systèmes fortement miniaturisés qui intègrent des microstructures mécaniques mobiles, vibrantes ou déformables. Ces microstructures sont actionnées et/ou détectées le plus souvent par voie électrostatique, piézoélectrique, électrothermique ou électromagnétique mais d'autres effets couplés avec la mécanique peuvent être mis en œuvre. D'une manière générale, la combinaison des MEMS avec l'optique est motivée par la grande précision et la faible valeur des déplacements ou des déformations possibles des micro/nano structures mécaniques et la possibilité de fabriquer de manière collective des composants, des capteurs ou des systèmes optiques ajustables avec une masse, un coût, une taille et un temps de réponse réduits. Ceci permet par exemple la réalisation de matrices de MOEMS.

La combinaison de l'optique avec les MEMS a démarré dans les années 80 avec comme développement marquant les matrices de micro-miroirs digitaux qui ont été commercialisés par Texas Instrument à partir du début des années 90. Initialement, les microsystèmes électromécaniques optiques (appelés MEMS optiques) visaient généralement le contrôle spatial de la lumière, notamment le balayage et la commutation de faisceaux optiques pour l'affichage et les télécommunications optiques, et reposaient sur le microusinage de surface et/ou de volume du silicium avec une intégration monolithique du MEMS, souvent associée à des composants optiques macroscopiques. Depuis le milieu des années 90 , le sigle MOEMS (microsystèmes optoélectromécaniques) a été introduit pour signifier la combinaison des MEMS avec des composants micro-optiques, mais les deux appellations (microsystèmes électromécaniques optiques et MOEMS) sont très souvent utilisées sans distinction. D'autres technologies fondées sur les semiconducteurs III-V, au milieu des années 90 , puis les polymères ont alors été progressivement développées et d'autres voies d'intégration des systèmes optiques ont commencé à être explorées comme par exemple les micro-bancs optiques, les micro-instruments optiques intégrés $3 D$, les technologies de report ou l'introduction de la micro-fluidique.

Une dizaine d'équipes de recherche académique françaises participent à cette évolution des MEMS optiques et des MOEMS en produisant régulièrement des résultats conformes à l'état de l'art. À l'Institut d'électronique fondamentale (IEF), plusieurs projets de réalisation de microsystèmes optiques/MOEMS intégrés 3D 
sont en cours. Ils sont décrits dans les paragraphes suivants.

\section{Matrices de filtres Fabry Péroł accordables pour l'imagerie spectrale}

L'imagerie spectrale consiste à acquérir plusieurs images de la même scène dans des bandes spectrales différentes. Ces images sont ensuite traitées pour améliorer la détection d'objets selon leur signature spectrale. Cette technique d'imagerie trouve des applications dans les domaines de la défense, de la médecine, et de la biologie. L'imagerie hyper-spectrale, qui exploite plusieurs dizaines à une centaine d'images haute résolution enregistrées successivement dans différentes plages de longueur d'ondes, fournit de nombreuses informations mais nécessite des temps d'acquisition et de traitement élevés. Une alternative permettant une observation en temps réel est l'imagerie multi-spectrale où plusieurs images de plus faible résolution sont enregistrées simultanément dans un nombre limité de bandes sélectionnées (figure 1). En exploitant une matrice de filtres accordables dans une large bande, ces bandes pourraient être adaptées dynamiquement à la scène. L'objectif des travaux de I'IEF, menés dans le cadre d'une action coordonnée de la SAGEM et impliquant I'IOGS (Institut d'optique Graduate School), le LPN (Laboratoire de photonique et des nanostructures) et l'Onera (Office national d'études et recherches aérospatiales), vise la réalisation d'une telle matrice de filtres accordables afin de réaliser une caméra multi-spectrale.

Une étude réalisée par l'IOGS a démontré qu'avec quatre filtres Fabry-Pérot de finesse modérée (voisine de 5) et accordables dans le domaine visible-proche infrarouge (550-850 $\mathrm{nm}$ ), des résultats satisfaisants pourraient être obtenus. De nombreux exemples de filtres individuels Fabry Pérot accordables avec des miroirs de petite taille ont été publiés pour le domaine proche infrarouge mais très peu dans le domaine visible. Le challenge pour l'imagerie multi-spectrale est la réalisation d'une matrice de filtres Fabry-Pérot de taux de remplissage élevé (>50\%), fonctionnant dans une large bande spec-

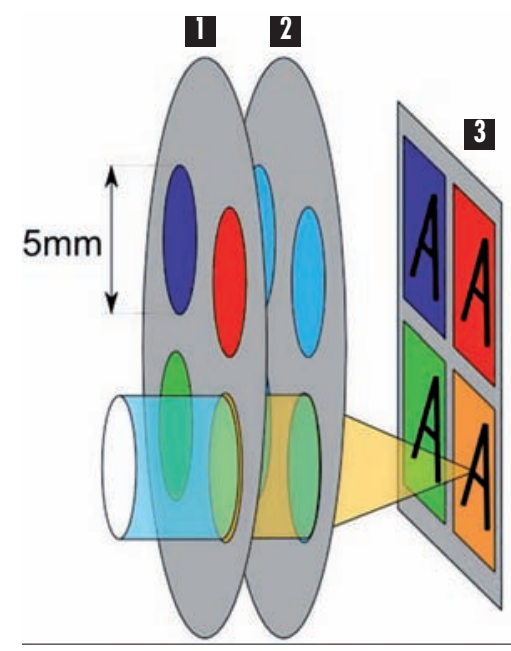

Figure 1. Principe d'un imageur multi-spectral adaptatif. (1) Matrice de filtres Fabry-Pérot accordables. (2) Matrices de lentilles. (3) Capteur d'images. trale, de grand diamètre $(5,2 \mathrm{~mm})$ et de transmission élevée (>80 \%). La configuration retenue à l'IEF pour la réalisation de ces filtres (figure 2) repose sur l'assemblage vertical de trois wafers : un wafer de verre sur lequel est réalisé un miroir diélectrique fixe, un wafer de silicium intermédiaire intégrant un miroir diélectrique mobile, et un wafer de verre utilisé pour l'encapsulation du filtre [1]. Un alignement par interférométrie Moiré est prévu pour atteindre une précision submicronique. Le miroir mobile est réalisé en intégrant une membrane multicouche (quatre paires $\mathrm{Si}_{x} \mathrm{~N}_{\mathrm{y}} / \mathrm{SiO}_{2}$ ) sur un cadre de silicium suspendu par trois ressorts. Le gap optique entre les miroirs est ajusté en translation et en inclinaison par actionnement électrostatique à l'aide de trois paires d'électrodes qui ont été conçues pour permettre également un contrôle intégré et un asservissement du gap d'air physique avec une précision meilleure que +/-15 $\mathrm{nm}$. La réalisation de cette matrice de filtres, qui nécessite pas moins de 11 niveaux de masquage, est très complexe. Elle nécessite d'obtenir des membranes multicouches de grand diamètre $(5,2 \mathrm{~mm})$, de très bonne planéité $(<30 \mathrm{~nm})$, de faible rugosité et de réflectivité homogène et peu variable dans une large bande spectrale. La figure 3 montre que la réalisation de telles membranes est en bonne voie. Il faut aussi contrôler des gaps physiques d'air très faibles entre les miroirs (jusqu'à $200 \mathrm{~nm}$ ) et assurer une bonne homogénéité des gravures profondes de silicium dans des ouvertures de tailles très variables. Ces différents
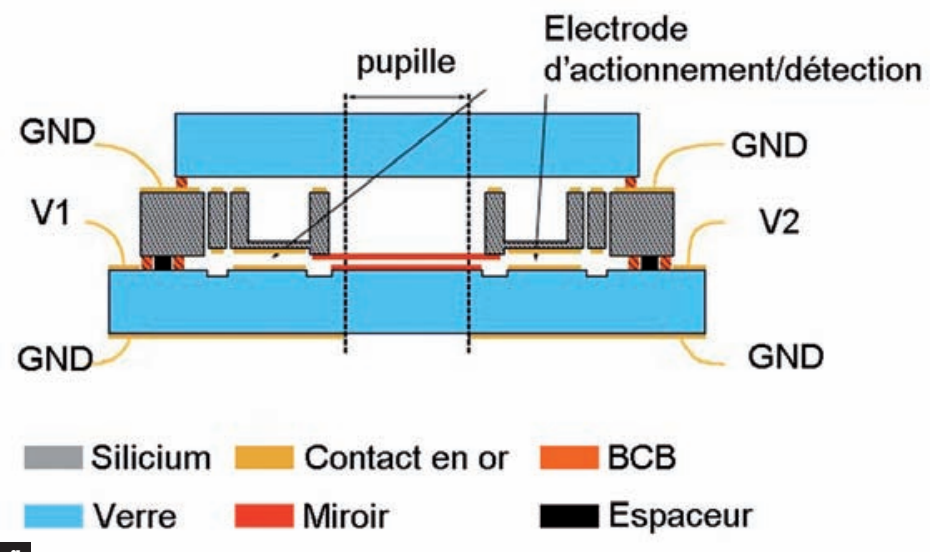

Figure 2. Configuration des filtres accordables Fabry-Pérot. (a) Schéma en coupe d'un filtre Fabry-Pérot. (b) Dessin en perspective des miroirs mobiles.

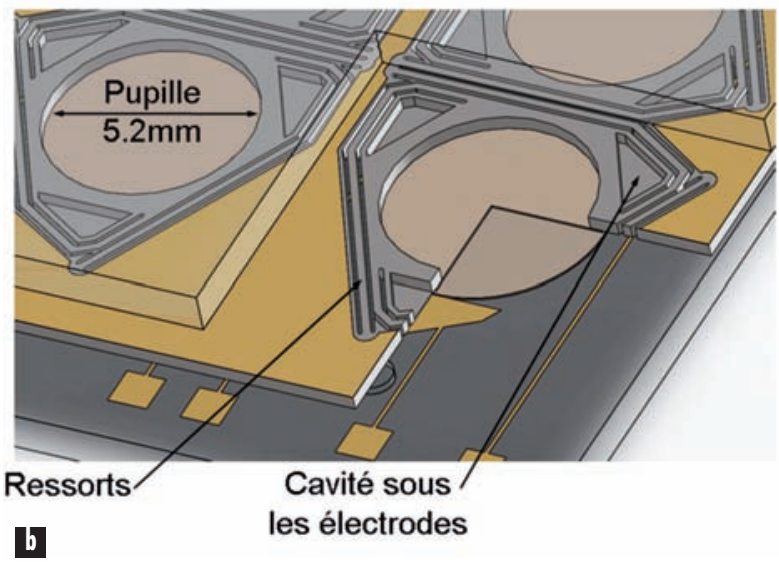




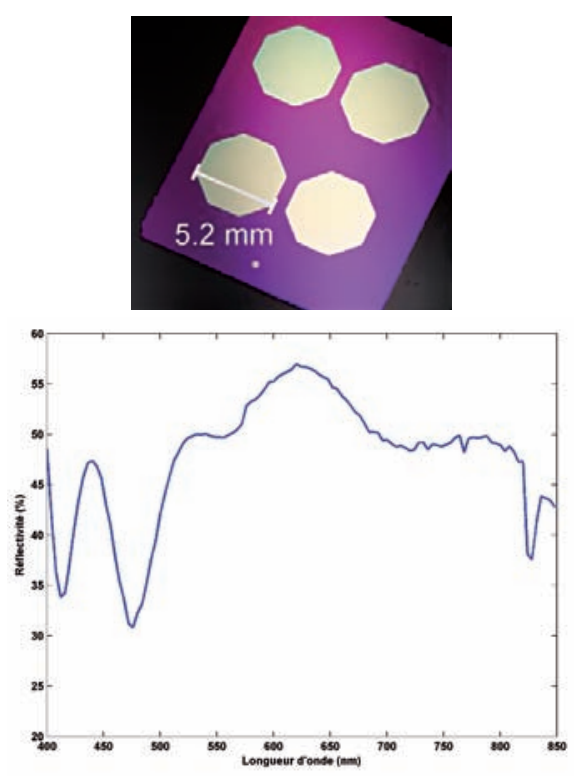

Figure 3. Image de membranes multicouches et réflectivité spectrale mesurée avant optimisation.

verrous technologiques ont été étudiés en détail et la réalisation complète d'une matrice de filtres est en cours.

\section{Micro-interféromètres Mirau larges accordables}

La microscopie interférométrique en lumière blanche ou monochrome est une technique très répandue pour la mesure plein champ, avec une résolution verticale sub-nanométrique, de profils de surface $3 \mathrm{D}$ et des déformations, déplacements et vibrations de microstructures mécaniques [2]. Elle repose sur l'utilisation d'objectifs interférométriques Michelson, Mirau ou Linnik et une translation de résolution nanométrique de l'échantillon ou de l'objectif pour obtenir des interférogrammes avec des différences de chemin optiques variables. Les objectifs interférométriques Mirau (figure 4), en raison de leur configuration planaire, sont ceux qui se prêtent le mieux à la miniaturisation. Bien que le premier interféromètre Mirau partiellement intégré ait été réalisé dès 1990, seulement quelques interféromètres Mirau et objectifs interférométriques Mirau partiellement ou complètement intégrés ont ensuite été proposés pour un fonctionnement en lumière monochrome dans le domaine visible, UV ou DUV.

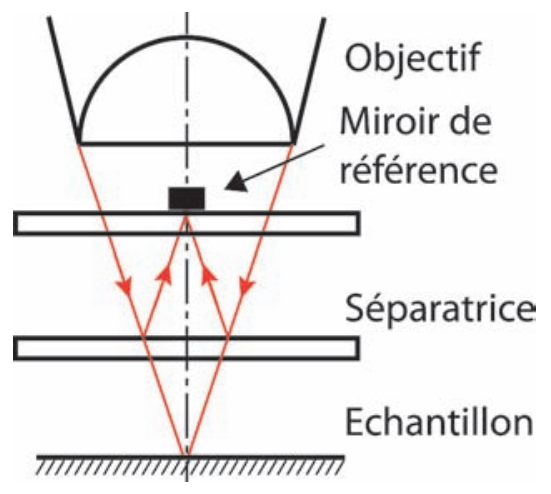

Figure 4. Schéma de principe d'un objectif interférométrique de type Mirau.

L'intérêt pour des objectifs interférométriques intégrés a été relancé récemment pour la caractérisation de grandes surfaces, ou celle de nombreux micro-dispositifs ou de micro-objets sur une grande surface, pour laquelle une mesure parallélisée avec des matrices d'objectifs interférométriques accordables s'avère nécessaire. La mise en œuvre de cette idée que nous avions envisagée fin 2003 pour réaliser un contrôle sur wafer de MEMS, a été reconsidérée récemment dans notre laboratoire. L'institut Femto-ST a déjà obtenu des résultats intéressants en démontrant la possibilité de réaliser des matrices de micro-objectifs interférométriques Mirau monochromes fonctionnels.

L'objectif que nous visons est de franchir des étapes supplémentaires en réalisant des matrices de micro-interféromètres Mirau pouvant fonctionner en mode

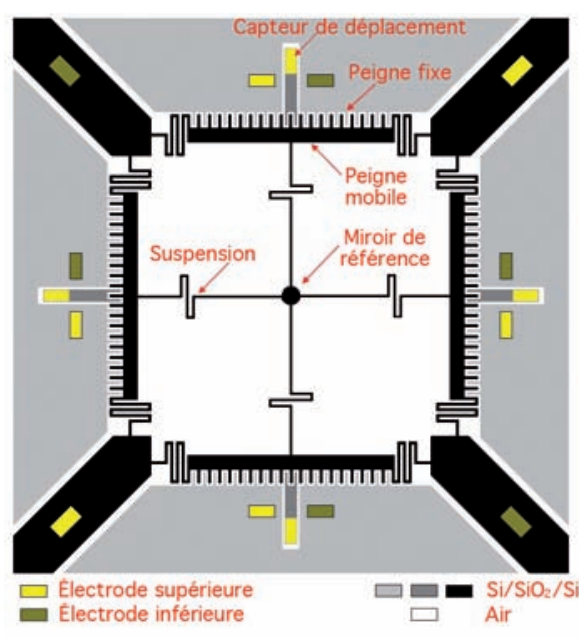

Figure 5. Vue de dessus du miroir translatable d'un interféromètre Mirau intégré.

\section{Micro positionnement pour l'usinage laser}

Les contrôleurs, les platines et les systèmes Aerotech augmentent la précision et le maximisant les temps de bon fonctionnement. PRO Series

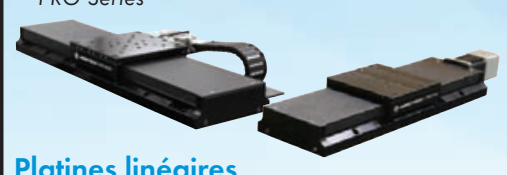

Platines linéaires

- Courses de 50 à $1500 \mathrm{~mm}$

- Vitesse jusqu'à $2 \mathrm{~m} / \mathrm{s}$

- Conçus avec des joints latéraux et un capot de protection en tôle

- Entrainement à vis à bille ou à moteur linéaire

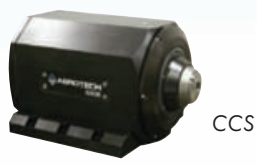

Rotation à entrainement direct

- Mandrin à serrage et desserrage pneumatique

- Collet captif minimise les défauts axiaux pendant le serrage/desserrage

- Ouverture central pour alimentation

- Moteur brushless et codeur dans l'axe

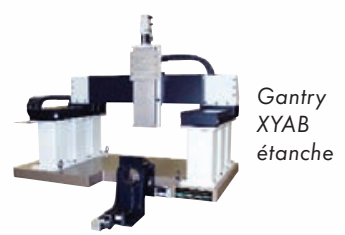

Systèmes Gantry

à moteurs linéaires

- Vitesse de $3 \mathrm{~m} / \mathrm{s}$ et accélération de $5 \mathrm{~g}$

- Précision exceptionnelle, haut rendement et grande productivité

- Versions étanches et options customisées pour votre application

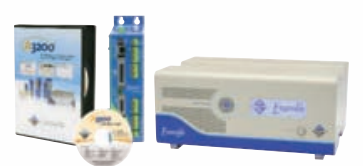

Plateformes contrôleurs avancés

- Mono et multiaxes

- Fonctions de contrôle avancées et software modulaire

- Environnement d'automation embarqué PLC

Télécharger notre brochure de solution pour process laser sur www.aerotech.com

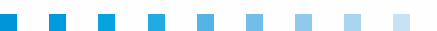
Dedicated to the AEROTECH ${ }^{\infty}$ Science of Motion AEROTECH $H^{\infty}$ Aerotech France, BP 70043 45702 Villemandeur Cedex Ph: +33 238970830 Email: ventes@aerotech.com

www.aerotech.com 

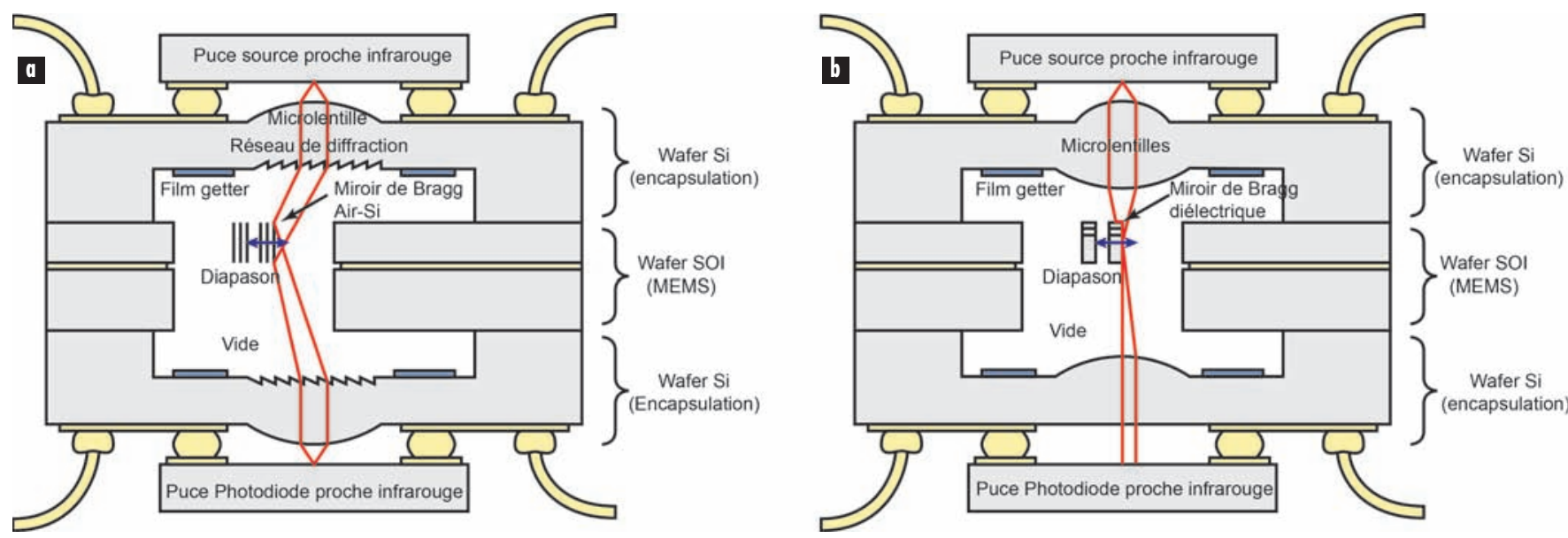

Figure 6. Schémas de principe de résonateurs en silicium encapsulés sous vide avec transduction opto-mécanique. (a) Actionnement par pression de radiation et détection par déflexion de faisceau. (b) Actionnement par gradient de champ optique et détection par obscuration de faisceau.

monochrome ou en lumière faiblement cohérente, dans les domaines visible ou proche infrarouge, et accordables en phase. Le micro-interféromètre sera constitué d'un miroir suspendu et actionné en translation à l'aide de peignes électrostatiques verticaux (figure 5) et d'une membrane séparatrice multicouches de même technologie que celles de la figure 3.

\section{Micro-résonateurs avec transduction opto-mécanique}

Le coefficient de qualité, qui qualifie les pertes d'énergie vibratoire et la finesse d'une résonance, est une caractéristique majeure des résonateurs mécaniques. Sa valeur a un impact direct sur la résolution, la sensibilité et la stabilité des oscillateurs et des micro-capteurs résonants. Les micro-résonateurs basse fréquence en silicium ou en GaAs monocristallin peuvent présenter des facteurs de qualité sous vide très élevé $\left(>10^{5}\right)$ si les résonateurs sont dimensionnés de façon appropriée et si les pertes d'énergie via les ancrages sont minimisées $[3,4]$. Le coefficient de qualité des micro-résonateurs est cependant fortement dégradé lorsqu'ils sont intégrés dans des microsystèmes électromécaniques en raison de la nécessité de les recouvrir d'électrodes pour réaliser l'actionnement et la détection des vibrations. De plus la transduction électromécanique impose un packaging sous vide complexe pour permettre le passage des connexions électriques. Pour conserver le coefficient de qualité sous vide élevé des résonateurs monocristallins, la solution que nous étudions dans le cadre d'un projet de la fondation EADS en collaboration avec l'Onera et I'ESIEE repose sur un actionnement optique par pression de radiation (figure 6a) ou par gradient de champ optique (figure $6 b$ ) et une détection du mouvement par déflexion laser (figure 6a) ou par obscuration de faisceau (figure 6b). En utilisant un faisceau lumineux proche infrarouge modulé en intensité, l'actionnement et la détection peuvent être réalisés à travers l'encapsulation sous vide sur wafer et, de plus, sans échauffement du micro-résonateur. La mise en forme du faisceau à l'entrée et à la sortie est réalisée en intégrant des éléments micro-optiques dans les wafers du packaging. Le déphasage temporel de $90^{\circ}$ entre l'excitation et la réponse mécanique permet d'utiliser le même faisceau pour l'actionnement et la détection. Les résonateurs choisis pour les tests sont des doubles diapasons avec des miroirs de Bragg air-Si verticaux qui ont été réalisés à I'ESIEE par gravure ionique réactive profonde. Le montage d'un système optique permettant la mesure sous vide des vibrations hors plan et dans le plan de microrésonateurs, avec actionnement optique proche infrarouge, est en cours de finalisation pour la caractérisation des méthodes de transduction mécanique proposées. En parallèle, nous avons développé un procédé de fabrication de microlen- tilles en silicium de grande taille et développons le packaging sous vide intégrant ces microlentilles et/ou des réseaux de diffraction et des films de piégeage des gaz (getters) pour obtenir une pression faible $\left(<10^{-2} \mathrm{mbar}\right)$ et durable.

\section{Perspectives}

Les développements en cours pour les trois projets de MOEMS présentés ci-dessus devraient permettre à terme d'établir une filière de fabrication de MOEMS intégrés 3D qui pourra être mise à profit pour réaliser d'autres systèmes électromécaniques optiques.

\section{Références}

[1] H. Bertin, A. Bosseboeuf, P. Coste, J. Minet, J. Taboury, M. Péalat, N. Roux. Design of a micromachined tunable fabry Pérot filter array for multispectral snapshot imaging. Optro 2012 Symposium, 8-12 février 2012, Paris

[2] A. Bosseboeuf, S. Petitgrand. Interference microscopy techniques for microsystem characterization. In: Optical inspection of Microsystems, W. Osten (Ed.), CRC Press, pp. 217-244, 2007

[3] B. Le Foulgoc, T. Bourouina, O. Le Traon, A. Bosseboeuf, F. Marty, C. Breluzeau, J.P. Grandchamp, S. Masson. Highly decoupled single-crystal resonateirs: an approach for the intrinsic quality factor. J. Micromech. Microeng. 16, S45S43, 2006

[4] I. Roland, S. Masson, O. Ducloux, O. La Traon, A. Bosseboeuf. GaAs-based tuning fork microresonators: a first step towards gaAs-based coriolis 3-axis micro-vibrating gyro (3 axis $\mu \mathrm{CVG}$ ). Sensors and actuators A 172, 204-211, 2011 